\title{
Statistical Mechanical Perturbation Theory of Solid-Vapor Interfacial Free Energy
}

\author{
V.I. Kalikmanov* $1,2,3$, R. Hagmeijer ${ }^{2}$, C.H. Venner ${ }^{2}$ \\ ${ }^{1}$ Twister Supersonic Gas Solutions, Einsteinlaan 20, 2289 CC, \\ Rijswijk; ${ }^{2}$ Engineering Fluid Dynamics, University of Twente, \\ P.O. Box 217, 7500 AE, Enschede; ${ }^{3}$ Department of Geosciences, \\ Delft University of Technology, Stevinweg 1, 2628 CN, Delft, Netherlands
}

(Dated: March 13, 2017)

\begin{abstract}
The solid-vapor interfacial free energy $\gamma_{s v}$ plays an important role in a number of physical phenomena, such as adsorption, wetting and adhesion. We propose a closed form expression for the orientation averaged value of this quantity using a statistical mechanical perturbation approach developed in the theory of liquids. Calculations of $\gamma_{s v}$ along the sublimation line for systems characterized by truncated and shifted Lennard-Jones potential are presented. Within the temperature range studied - not far from the triple point - model predictions are in good agreement with molecular dynamics simulations. At the triple point itself the model yields interfacial tensions between the three coexisting phases - solid-vapor, liquid-vapor and solid-liquid. The latter is obtained by means of Antonow's rule. All three triple point values perfectly agree with simulation results.
\end{abstract}

\section{INTRODUCTION}

The solid-vapor interfacial free energy $\gamma_{s v}$ plays an important role in a number of physical phenomena, such as wetting, nucleation, adsorption and surface wear ${ }^{1}{ }^{3}$. In particular, $\gamma_{s v}$ enters the Dupré-Young equation describing a liquid droplet resting on a rigid flat surface of solid exposed to vapor ${ }^{4}$

$$
\gamma_{s v}=\gamma_{s l}+\gamma_{l v} \cos \theta
$$

where $\gamma_{s l}$ and $\gamma_{l v}$ are the solid-liquid and liquid-vapor interfacial free energies, respectively, and $\theta$ is the equilibrium contact angle. The Dupré-Young equation plays a key role in the theory of wetting phenomena. If the three free energies are known, the wetting state of the fluid follows directly. When $\gamma_{s v}<\gamma_{s l}+\gamma_{l v}$, a droplet with finite contact angle $\theta$ minimizes the free energy of the system, resulting in partial wetting. On the other hand, if $\gamma_{s v}=\gamma_{s l}+\gamma_{l v}$, the contact angle is zero resulting in complete wetting when a macroscopic liquid layer covers the whole solid surface. In nucleation studies the interfacial free energy is one of the key parameters determining the nucleation barrier and nucleation rate $^{2}$.

A specific feature of interfaces involving solids is that the interfacial free energy is not equal to the interfacial tension (stress) $\tau_{s v}$ (being the average of the lateral components of the surface stress tensor). The two quantities are related by ${ }^{5}$

$$
\tau_{s v}=\gamma_{s v}+A \frac{\mathrm{d} \gamma_{s v}}{\mathrm{~d} A}
$$

where $A$ is the surface area. The difference between $\tau_{s v}$ and $\gamma_{s v}$ stems from the fact that unlike a fluid, crystal is able to support stress. For fluids, $\mathrm{d} \gamma_{s v} / \mathrm{d} A=0$ because

\footnotetext{
*E-mail: Vitaly.Kalikmanov@twisterbv.com
}

the fluid-fluid interface is insensitive to strain. Solid interfaces are in general sensitive to strain upon compression or stretching yielding the inequality $\tau_{s v} \neq \gamma_{s v}$.

Among the four quantities entering Eq. (1) only two - $\gamma_{l v}$ and $\theta$ - are directly accessible in experiment, while it is still difficult to measure $\gamma_{s v}$ and $\gamma_{s l}$. The solidliquid and solid-vapor interfacial free energies were measured for a very limited number of substances ${ }^{6-8}$. That is why a number of theoretical and simulation efforts have been undertaken to calculate these quantities in the absence of experimental means. Theoretically, the primary approach was the use of the density functional theory applied for a number of simple systems, such as hard spheres and Lennard-Jones systems ${ }^{9}{ }^{11}$. The solid-liquid interfacial free energy was studied in molecular dynamics (MD) simulation for hard spheres ${ }^{12,13}$, soft spheres $^{14}$, Lennard-Jones fluids and their mixtures ${ }^{15,16}$.

The important feature of solid-vapor interfacial free energy is that it is a property of the solid and as such does not depend on the particular liquid wetting it (partially or completely). This is the reason that $\gamma_{s v}$ is frequently referred to as the solid surface free energy. Molecular dynamics simulations of solid-vapor interfaces were pioneered by Broughton and Gilmer ${ }^{17},{ }^{18}$. They considered a two-phase system consisting of a slab of a crystal interacting with a slab of vapor. Performing simulations in the two bulk systems - solid and vapor and the two-phase vapor-solid system, they calculated the surface excess entropy $S^{\text {s }}$ at temperature $T$ from numerical integration of the surface excess energy $E^{\mathrm{s}}$ and stress data from the zero Kelvin limit

$$
S^{\mathrm{s}}=S_{0}^{\mathrm{s}}+\int_{0}^{T}\left(\mathrm{~d} E^{\mathrm{s}}-\tau_{s v} \mathrm{~d} A\right) / T
$$

where $S_{0}^{\mathrm{s}}$ is the zero Kelvin value obtained from lattice dynamics. The quantities $E^{\mathrm{S}}(T)$ and $\tau_{s v}(T)$ were derived from polynomial fits of the bulk and two-phase simulation data. Applying the concept of the Gibbs equimolar dividing surface, the solid-vapor interfacial free energy is 
found from the relationship (see, e.g., ${ }^{19}$ )

$$
\gamma_{s v}=\frac{E^{\mathrm{s}}-T S^{\mathrm{s}}}{A}
$$

Smith and Lynden-Bell ${ }^{20}$ proposed an alternative method in which instead of thermodynamic integration over the temperature one performs simulations at a single temperature of interest by varying the interaction between crystal slabs from full to no interactions. This method was recently developed by Modak, Wyslouzil and Singer ${ }^{21}$ who applied it for calculations of the vapor-solid surface free energy of $n$-alkanes. The solid surface free energy plays an important role in material science. The temperature dependence of $\gamma_{s v}$ for $C u$ was studied in Monte Carlo simulations ${ }^{22},{ }^{23}$, where it was found that $\gamma_{s v}$ decreases with temperature.

In the present paper we propose an alternative analytical route to calculate $\gamma_{s v}$ originating from the liquid state theory. In 1949 Kirkwood and Buff ${ }^{24}$ derived an exact expression for the interfacial free energy of a planar liquid-vapor interface in a system of pairwise interacting particles:

$\gamma_{l v}=\frac{1}{4} \int_{-\infty}^{+\infty} \mathrm{d} z_{1} \int \mathrm{d} \mathbf{r}_{12}\left(r_{12}-\frac{3 z_{12}^{2}}{r_{12}}\right) u^{\prime}\left(r_{12}\right) \rho^{(2)}\left(\mathbf{r}_{1}, \mathbf{r}_{2} ; \rho\right)$

Here $\rho^{(2)}\left(\mathbf{r}_{1}, \mathbf{r}_{2} ; \rho\right)$ is a pair distribution function of the two-phase system, describing correlations between particles located at the points $\mathbf{r}_{1}$ and $\mathbf{r}_{2}, \mathbf{r}_{12}=\mathbf{r}_{1}-\mathbf{r}_{2} ; \rho$ is the number density, ${ }^{\prime}=\mathrm{d} / \mathrm{d} r_{12}$, inhomogeneity is in the $z$ direction. Note, that the original derivation of $\gamma_{l v}$ in Ref. ${ }^{24}$ was based on the microscopic pressure tensor, $\hat{\mathbf{p}}$, considerations. Meanwhile, as found by Schofield and Henderson $^{25}$, the form of $\hat{\mathbf{p}}$ is not unique. The same result (2) can be derived avoiding the ambiguity in $\hat{\mathbf{p}}$ using general statistical thermodynamic considerations (see, e.g. ${ }^{26},{ }^{19}$ ).

Application of Eq. (2) for $\gamma_{l v}$ requires the knowledge of $\rho^{(2)}$ and $\rho$. Unfortunately, no practicable and exact routes exist to determine these functions in the interfacial domain from the knowledge of $u(r)$ only. If the density difference between the phases is substantial, which is the case of vapor-liquid interface far from the critical point, one can resort to the Fowler approximation in which the physical transition zone is shrunk to a mathematical surface of density discontinuity. Equation (2) then results $\operatorname{in}^{26}$

$$
\gamma_{l v}=\frac{\pi}{8}\left(\rho^{l}\right)^{2} \int_{0}^{\infty} r^{4} u^{\prime}(r) g\left(r ; \rho^{l}, T\right) \mathrm{d} r
$$

where $\rho^{l}(T)$ is the number density of the bulk liquid and $g\left(r ; \rho^{l}, T\right)$ is the pair correlation function in the bulk liquid.

The present paper is based on the observation that similar considerations are applicable to the solid-vapor interface not far from the triple point $T_{T P}$. Indeed, as found in MD simulations of Broughton and Gilmer ${ }^{18}$, the surface free energy $\gamma_{s v}$ is nearly isotropic within $20 \%$ of the triple point (as opposed to the surface stress $\tau_{s v}$ which remains highly anisotropic at all temperatures). It is also obvious, that the density difference between solid and vapor is high which justifies the use of the Fowler approximation. We construct a simple theoretical model for the orientation-averaged surface free energy of a crystal not far $T_{T P}$. The model is based on the ideas of the statistical mechanical perturbation approach developed in the theory of liquids (see e.g., ${ }^{19}$ ) which was applied earlier to calculation of the vapor-liquid interfacial free energy ${ }^{27}$. Within the framework of the perturbation approach the intermolecular interaction potential $u(r), r$ being the intermolecular separation, is decomposed into the reference $u_{0}(r)$ and perturbative $u_{1}(r)$ part: $u(r)=u_{0}(r)+u_{1}(r)$. Consequently, the system under study is decomposed into a reference model, characterized by a reference potential $u_{0}$ and the same density and temperature as the original system, and a perturbation. Properties of the reference model are assumed to be known to appreciable accuracy. The thermodynamics of the full system is obtained by appropriate averaging of the perturbation over the reference model. The peculiar thing about application of this approach to condensed system is that the reference model must be nonideal. In most cases it is a hard-sphere system with an appropriately chosen effective diameter. The reason for the success of perturbation theories is that the structure of a liquid is determined primarily by the repulsive (hard-core) part of the interaction, while the attractive part provides a uniform background potential in which the molecules move. Within the first-order (meanfield) approach the free energy expansion in $\beta \epsilon$, where $\epsilon$ is the depth of the interaction potential $u(r), \beta=1 / k_{\mathrm{B}} T$, $T$ is the temperature, $k_{\mathrm{B}}$ is the Boltzmann constant, is truncated at the first order term which represents the average contribution of attractive interactions to the free energy. The higher-order terms take into account effects of changing structure resulting from the perturbation. If the density is high, as in liquids (and solids), these changes in structure become increasingly difficult since particles are closely packed. Therefore, at high densities the higher-order perturbation terms become small and the perturbation expansion converges rapidly even if $\beta \epsilon$ is not small. This is one of the main reasons for the success of the mean-field perturbation approach in the theory of liquids.

Applying the mean-field perturbation approach to solids, it is necessary to bear in mind that by construction the free energy expansion in $\beta \epsilon$ remains a high-temperature approximation and diverges in the zero Kelvin limit. Therefore, the theory discussed in the present paper is valid for temperatures not too far from the triple point. Based on MD simulations ${ }^{18}$ the temperature range for applicability of the model can be set equal to $0.8 T_{T P}<T \leq T_{T P}$.

The paper is organized as follows. In Sect. II we formulate the framework of statistical mechanical perturbation approach to describe the surface-vapor phase equilibrium 
along the sublimation line. In Sect. III we present the results of calculations of the interfacial free energy for the system described by the truncated and shifted LJ potential and compare our theoretical predictions with available simulation data. Separate attention is paid to the triple point in which the theory allows calculation of all three interfacial free energies - vapor-solid, liquid-vapor and liquid-solid. We finish by presenting our conclusions.

\section{PERTURBATION APPROACH TO INTERFACIAL FREE ENERGY}

To calculate the solid-vapor interfacial free energy

$$
\gamma_{s v}=\left(\frac{\partial \mathcal{F}}{\partial A}\right)_{N, V, T},
$$

where $\mathcal{F}$ is the Helmholtz free energy of the two-phase solid-vapor system, containing $N$ molecules in the volume $V$, we follow the same lines as in the theory of vaporliquid interface ${ }^{26}$. We assume that

(i) the intermolecular interaction energy is pairwise additive,

(ii) the interaction potential $u(r)$ (where $r$ is the intermolecular separation) is spherically symmetric, and

(iii) inhomogeneity is in the $z$ direction.

The quantity $\gamma_{s v}$, discussed in the present paper, is the orientation averaged plane layer property. Our starting point is the Fowler approximation (3) written for the solid-vapor system

$$
\gamma_{s v}=\frac{\pi}{8}\left(\rho^{s}\right)^{2} \int_{0}^{\infty} r^{4} u^{\prime}(r) g\left(r ; \rho^{s}, T\right) \mathrm{d} r
$$

where $\rho^{s}(T)$ is the number density of the bulk solid and $g\left(r ; \rho^{s}, T\right)$ is the pair correlation function in the bulk solid. Let us introduce the (orientation-averaged) cavity function in the solid phase

$$
y\left(r ; \rho^{s}, T\right)=g\left(r ; \rho^{s}, T\right) \mathrm{e}^{\beta u(r)}
$$

The important feature of $y(r)$ is that it remains continuous for all values of $r$ as opposed to $g(r)$ which can have a finite jump for discontinuous intermolecular potentials, e.g. for hard spheres ${ }^{19}$. Using (6), Eq.(5) can be written as:

$$
\gamma_{s v}=\frac{\pi}{8}\left(\rho^{s}\right)^{2} k_{\mathrm{B}} T\left[\alpha_{1}(T)+\alpha_{2}\left(\rho^{s}, T\right)\right],
$$

where

$$
\begin{aligned}
& \alpha_{1}(T)=-\int_{0}^{\infty} r^{4} f^{\prime}(r) \mathrm{d} r \\
& \alpha_{2}\left(\rho^{s}, T\right)=-\int_{0}^{\infty} r^{4} f^{\prime}(r)\left[y\left(r ; \rho^{s}, T\right)-1\right] \mathrm{d} r
\end{aligned}
$$

Here $f=\mathrm{e}^{-\beta u}-1$ is the Mayer function of the potential $u(r)$. Integration of (8) by parts yields

$$
\alpha_{1}(T)=4 \int_{0}^{\infty} f(r) r^{3} \mathrm{~d} r
$$

The temperature-dependent quantity $\alpha_{1}(T)$ is thus calculated straightforwardly for a given interaction potential $u(r)$.

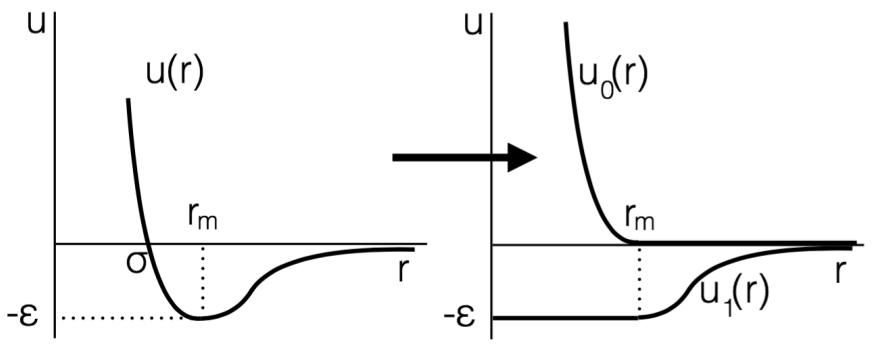

FIG. 1: Weeks-Chandler-Andersen decomposition of a typical interaction potential $u(r) ; u_{0}(r)$ - reference part, $u_{1}(r)$ perturbation.

In what follows we focus on evaluation of $\alpha_{2}\left(\rho^{s}, T\right)$. A typical interaction potential $u(r)$ is characterized by a short-range repulsive and a long-range attractive part as schematically depicted in Fig. 1. Using the WeeksChandler-Andersen (WCA) perturbation theory ${ }^{28}$, we decompose $u(r)$ into the reference $u_{0}(r)$ and perturbative $u_{1}(r)$ part: $u(r)=u_{0}(r)+u_{1}(r)$ with

$$
\begin{gathered}
u_{0}(r)=\left\{\begin{array}{l}
u(r)+\epsilon \text { for } r<r_{m}, \\
0 \text { for } r \geq r_{m},
\end{array}\right. \\
u_{1}(r)=\left\{\begin{array}{l}
-\epsilon \text { for } r<r_{m}, \\
u(r) \text { for } r \geq r_{m},
\end{array}\right.
\end{gathered}
$$

where $\epsilon$ is the depth of the potential and $r_{m}$ is the corresponding value of $r: u\left(r_{m}\right)=-\epsilon$. Figure 2 schematically shows the behavior of the quantities entering Eq. (9) the derivative of the Mayer function $f^{\prime}(r)$ and the cavity function $y(r)$ - for a typical interaction potential $u(r)$.

In the domain $r<r_{m}$ the function $f^{\prime}(r)$ has a sharp positive peak at some $r_{0}$ close to $r_{m}$ whereas $y(r)$ monotonically decreases. In the domain $r>r_{m}$ the function $f^{\prime}(r)$ is negative and asymptotically tends to zero, whereas $[y(r)-1]$ oscillates about zero ${ }^{19}$. In view of these oscillations we set the upper limit of the integral in (9) equal to $r_{m}$.

Consider the domain $r<r_{m}$. Here from (11)

$$
f^{\prime}(r)=f_{0}^{\prime}(r) \mathrm{e}^{\beta \epsilon}, \quad r<r_{m}
$$

where $f_{0}(r)=\mathrm{e}^{-\beta u_{0}(r)}-1$ is the Mayer function of the reference system. By virtue of the perturbation approach we approximate (12) as

$$
f^{\prime}(r) \simeq f_{0}^{\prime}(r)(1+\beta \epsilon), \quad r<r_{m}
$$

In the same domain one can replace the function $y(r)$ by its repulsive counterpart $y_{0}(r)=g_{0}(r) \mathrm{e}^{\beta u_{0}(r)}$, because $y(r)$ and $y_{0}(r)$ are quite similar; here $g_{0}(r ; \rho, T)$ is the pair 


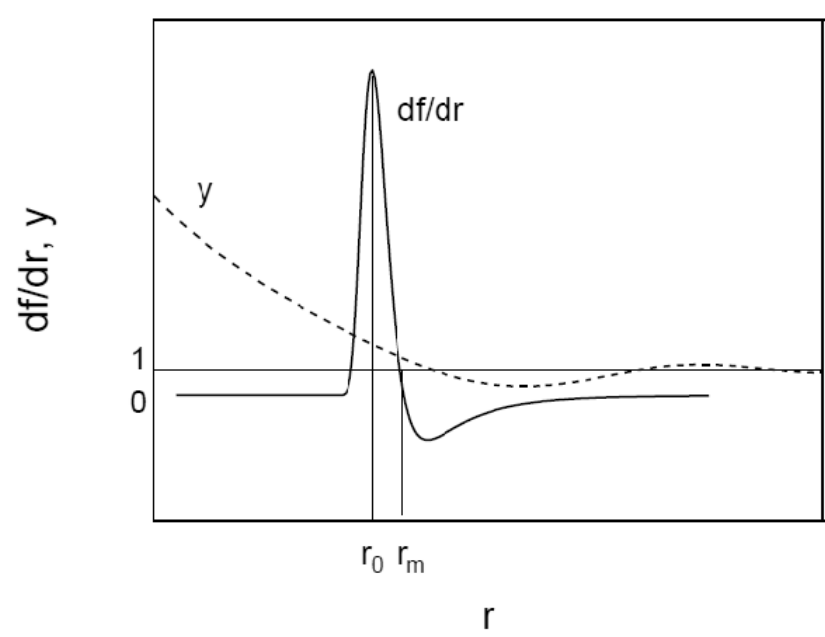

FIG. 2: Behavior of the derivative of the Mayer function $f^{\prime}(r)$ and the cavity function $y(r)$ for a typical interaction potential $u(r)$.

correlation function of the reference system. Equation (9) takes the form

$$
\alpha_{2} \simeq-\int_{0}^{r_{m}} r^{4} f_{0}^{\prime}(r)(1+\beta \epsilon)\left[y_{0}(r)-1\right] \mathrm{d} r
$$

Function $f_{0}^{\prime}(r)$ has a sharp peak at the same $r_{0}<r_{m}$ as $f^{\prime}(r)$, and therefore the major contribution to the integral comes from the vicinity of $r_{0}$, where $y_{0}(r)$ behaves to first order as a straight line with a negative slope $\left(\frac{d y_{0}}{d r}\right)_{R}$ (see Fig. 2):

$$
y_{0}(r)=y_{0}(R)+\left(\frac{d y_{0}}{d r}\right)_{R}(r-R)+\ldots
$$

Here $R$ is a point near $r_{0}$ which will be specified below. Substitution of (14) into (13) gives:

$$
\alpha_{2} \simeq a_{0}+a_{1}+a_{2}+\ldots
$$

where

$$
\begin{array}{r}
a_{0}=-\left[y_{0}\left(R ; \rho^{s}, T\right)-1\right] \int_{0}^{r_{m}} f_{0}^{\prime}(r) r^{4} \mathrm{~d} r \\
a_{1}=-\beta \epsilon\left[y_{0}\left(R ; \rho^{s}, T\right)-1\right] \int_{0}^{r_{m}} f_{0}^{\prime}(r) r^{4} \mathrm{~d} r \\
a_{2}=\left\{-\left(\frac{d y_{0}}{d r}\right)_{R}\right\} \int_{0}^{r_{m}} f_{0}^{\prime}(r) r^{4}(r-R) \mathrm{d} r
\end{array}
$$

One can see that $a_{1}<0$ and $a_{2}>0$ for all temperatures (for a reasonable choice of $R$ ). At low temperatures $a_{1}\left(\rho^{s}, T\right)$ and $a_{2}\left(\rho^{s}, T\right)$ compensate each other. This implies that it is plausible to set: $\alpha_{2} \approx a_{0}$, which after integration by parts yields

$$
\alpha_{2}=4\left[y_{0}\left(R ; \rho^{s}, T\right)-1\right] \int_{0}^{r_{m}} f_{0}(r) r^{3} \mathrm{~d} r
$$

Since the reference interaction is harshly repulsive, the cavity function $y_{0}(r)$ is fairly insensitive to a particular form of $u_{0}(r)$ and can be accurately mimicked by the cavity function of the hard-sphere system in the solid phase $y_{H S}^{s}\left(r ; \rho^{s}\right)$ with a suitably defined effective diameter $d_{H S}$. Within the WCA theory the effective diameter reads

$$
d_{H S}=\left[3 \int_{0}^{r_{m}}\left(1-\mathrm{e}^{\beta u_{0}(r)}\right) r^{2} \mathrm{~d} r\right]^{1 / 3}
$$

The quantity $y_{0}(R)$ is then replaced by the value of $y_{H S}^{s}$ at contact: $y_{0}\left(R ; \rho^{s}\right) \simeq y_{H S}^{s}\left(d_{H S} ; \rho^{s}\right) \equiv y_{d}^{s}$. Combining (7), (10) and (15), we obtain

$\gamma_{s v}=\frac{\pi}{2}\left(\rho^{s}\right)^{2} k_{\mathrm{B}} T\left[\int_{0}^{\infty} f(r) r^{3} \mathrm{~d} r+\left(y_{d}^{s}-1\right) \int_{0}^{r_{m}} f_{0}(r) r^{3} \mathrm{~d} r\right]$

The virial equation of state for hard spheres reads ${ }^{19}$

$$
\frac{p}{\rho k_{\mathrm{B}} T}=1+4 \eta_{H S} y_{d}^{s}
$$

where $p$ is the pressure, $\rho$ is the density, and $\eta_{H S}$ is the hard sphere packing fraction $\eta_{H S}=(\pi / 6) \rho d_{H S}^{3}$. From (18)

$$
y_{d}^{s}=\left(\frac{p}{\rho k_{\mathrm{B}} T}-1\right) /\left(4 \eta_{H S}\right)
$$

In the solid phase the compressibility factor of hard spheres is accurately described by Hall's equation of state $^{29}$

$$
\begin{gathered}
\frac{p}{\rho k_{\mathrm{B}} T}=\frac{3}{\xi}+2.557696+0.1253077 \lambda+0.176239 \lambda^{2} \\
-\quad 1.053308 \lambda^{3}+2.818621 \lambda^{4}-2.921934 \lambda^{5}+1.118413 \lambda^{6}
\end{gathered}
$$

where

$$
\xi=\frac{\eta_{c p}}{\eta_{H S}}-1, \quad \lambda=4\left(1-\frac{\eta_{H S}}{\eta_{c p}}\right)
$$

and $\eta_{c p}$ is the close packing; for the face-centered cubic (fcc) lattice ${ }^{30} \eta_{c p}=\pi \sqrt{2} / 6$.

\section{RESULTS AND DISCUSSION}

We illustrate the proposed model for the LennardJones (LJ) system with the interaction potential

$$
u_{L J}(r)=4 \epsilon\left[\left(\frac{\sigma}{r}\right)^{12}-\left(\frac{\sigma}{r}\right)^{6}\right]
$$

As is common we introduce the reduced variables, in which distances are measured in the units of $\sigma$ and energy - in the units of $\epsilon: r^{*}=r / \sigma, u^{*}=u / \epsilon$. The reduced temperature, number density and interfacial free energy become, respectively: $T^{*}=k_{\mathrm{B}} T / \epsilon, \rho^{*}=\rho \sigma^{3}, \gamma_{s v}^{*}=$ 
$\gamma_{s v} \sigma^{2} / \epsilon$. Equation (17) in reduced units reads (the superscript "*" is omitted):

$$
\gamma_{s v}=\frac{\pi}{2}\left(\rho^{s}\right)^{2} T\left[h_{1}+\left(y_{d}^{s}-1\right) h_{2}\right]
$$

with

$$
h_{1}(T)=\int_{0}^{\infty} f(r) r^{3} \mathrm{~d} r, \quad h_{2}(T)=\int_{0}^{r_{m}} f_{0}(r) r^{3} \mathrm{~d} r
$$

and $r_{m}=2^{1 / 6}$. Calculations of $\gamma_{s v}$ requires the solid density along the sublimation line. Solid-vapor coexistence in LJ systems was studied by van der Hoef ${ }^{31}$ who derived $\rho^{s}(T)$ from equation of state based on the free energy of the fcc LJ crystal:

$$
\rho^{s}(T)=\sum_{k=0}^{4} b_{k} T^{k}
$$

where $b_{0}=1.091, b_{1}=-0.134343, b_{2}=$ $-0.0950795, b_{3}=0.137215, b_{4}=-0.161890$. This expression is in excellent agreement with Monte Carlo simulations of Barroso and Ferreira ${ }^{32}$ based on the Einstein crystal method of Frenkel and Ladd ${ }^{33}$.

Broughton and Gilmer ${ }^{17,18}$ studied the surface free energy of the LJ crystal using the MD simulations for the truncated and shifted LJ potential

$$
u_{t L J}(r)=\left\{\begin{array}{l}
4\left[r^{-12}-r^{-6}\right]+C_{1}, \quad r \leq 2.3 \\
\left.C_{2} r^{-12}+C_{3} r^{-6}+C_{4} r^{2}+C_{5}, \quad 2.3<r<2 \mathfrak{2 3}_{3}\right) \\
0, \quad r \geq 2.5
\end{array}\right.
$$

where $C_{1}=0.016132, C_{2}=3136.6, C_{3}=-68.069, C_{4}=$ $-0.083312, C_{5}=0.74689$. [Note, that, as indicated earlier by Laird and Davidchak ${ }^{34}$, the sign of $C_{4}$ was incorrectly reported as positive in Brouhgton and Gilmer's original papers]. Such a choice provides continuity of the $u_{t L J}(r)$ and its first derivatives at $r=2.3$ and $r=2.5$. The triple point temperature for this potential was found to be $T_{T P}=0.617$.

The results for $\gamma_{s v}$ from Eqs.(20) -(22) using the same interaction potential $u_{t L J}(r)$ are shown in Fig. 3. Within the framework of the perturbation approach we are limited to the temperatures which are not too low, i.e. not far from the triple point. With this in mind calculations are performed for the temperature range $0.5 \leq T \leq$ 0.617. Also shown in Fig. 3 are MD simulation results of Ref. ${ }^{17}$ derived by thermodynamic integration from the zero temperature limit which are in good agreement with the model predictions. An important observation resulting from simulations ${ }^{17}$ is that in the temperature range $0.5<T<0.617$ the free energies of the crystal faces (111), (100), and (110) - are nearly identical and the differences are in the range of statistical uncertainty of the data, which justifies the use of the orientation averaged $\gamma_{s v}$ in the present model.

One can notice that the curvature of $\gamma_{s v}(T)$ is opposite to that of the simulation data. A possible reason for that can be the use in Eq. (17) of the cavity function of hard spheres $y_{H S}^{s}(r)$ instead of the cavity function $y_{0}(r)$ of the reference system. Unfortunately, the behavior of $y_{0}\left(r ; \rho^{s}, T\right)$ is not known. Meanwhile, as it is shown in the WCA theory ${ }^{28}$, the choice of the reference model according to the WCA decomposition (11) provides the approximate equality of $y_{0}$ and its hard-sphere counterpart $y_{H S}^{s}$ to the high degree of accuracy.

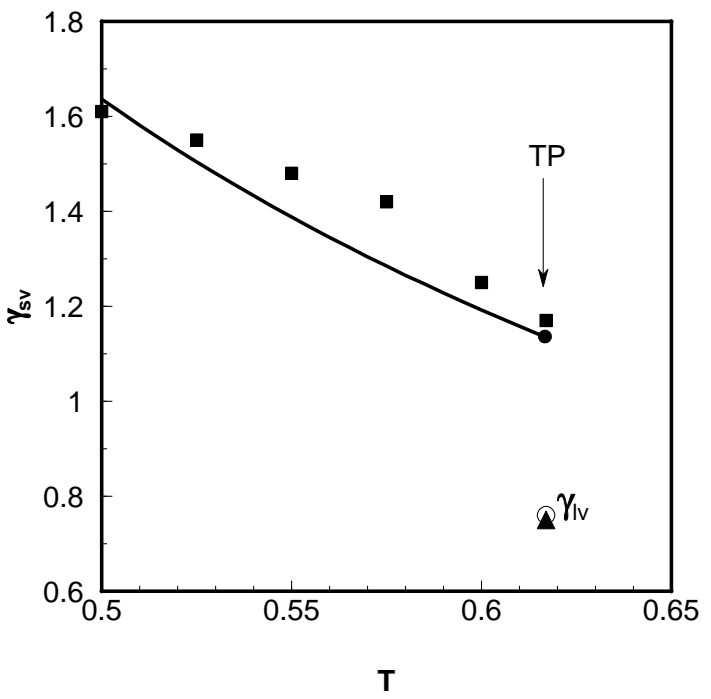

FIG. 3: Solid-vapor interfacial free energy of the truncated and shifted LJ system along the sublimation line. Solid line: present model; closed squares: MD simulations of Broughton and Gilmer ${ }^{17}$. Arrow indicates the triple point $T_{T P}=0.617$ found in MD simulations of ${ }^{17}$ and $\mathrm{MC}$ simulations of Barroso et al. ${ }^{32}$. Open circle: liquid-vapor surface free energy at the triple point $\gamma_{l v}\left(T_{T P}\right)$ predicted by the present model; closed triangle: $\gamma_{l v}\left(T_{T P}\right)$ found in MD simulations of Ref. ${ }^{17}$.

The same approach can be applied to vapor-liquid surface free energy $\gamma_{l v}$ not close to the critical point ${ }^{27}: \gamma_{l v}$ is then given by Eq.(20) in which $\rho^{s}$ should be replaced by the liquid density $\rho^{l}$ at vapor-liquid coexistence and $y_{d}^{s}$ - by the corresponding quantity for the liquid hard spheres, $y_{d}^{l}$.

$$
\gamma_{l v}=\frac{\pi}{2}\left(\rho^{l}\right)^{2} T\left[h_{1}+\left(y_{d}^{l}-1\right) h_{2}\right]
$$

The quantity $y_{d}^{l}$ is found from the highly accurate Carnahan-Starling theory (see e.g. ${ }^{19}$ ):

$$
y_{d}^{l}=\frac{4-2 \eta_{d}^{l}}{4\left(1-\eta_{d}^{l}\right)^{3}}
$$

where $\eta_{d}^{l}=(\pi / 6) \rho^{l} d_{H S}^{3}$ and the hard-sphere diameter $d_{H S}$ is given by Eq. (16).

It is instructive to calculate all interfacial free energies - vapor-solid, liquid-vapor and liquid-solid - at the triple point $T_{T P}$ where all three phases coexist. Davidchack 
and Laird ${ }^{16}$ performed MD simulations of crystal-melt interface for the same truncated and shifted LJ potential as used by Broughton and Gilmer. The coexistence liquid density at the triple point was found to be $\rho^{l}\left(T_{T P}\right)=$ 0.828. From Eqs. (24)-(25), we find $\gamma_{l v}\left(T_{T P}\right) \approx 0.760$ which is in perfect agreement with the simulation result of Ref. ${ }^{17} \gamma_{l v}^{M D}=0.75 \pm 0.05$.

Note, that the value of $\gamma_{l v}$ at the triple point found in Ref. ${ }^{27}$ was higher: $\gamma_{l v}\left(T_{T P}\right) \approx 1.4$ - which is due to the fact the coexistence liquid density in ${ }^{27}$ was calculated from the Song and Mason equation of state ${ }^{35}$. The latter is known to be accurate at higher temperatures but overestimates liquid densities (compared to simulations) as the triple point is approached ${ }^{35}$.

Assuming that liquid perfectly wets its own solid, we apply Antonow's rule 26

$$
\gamma_{s v}=\gamma_{s l}+\gamma_{l v}
$$

which corresponds to the Dupré-Young equation (1 ) for the case when liquid is spread as a film over the solidvapor interface yielding $\theta=0$. From (26) we determine the solid-liquid interfacial free energy $\gamma_{s l}$ at the triple point:

$$
\gamma_{s l}\left(T_{T P}\right)=\gamma_{s v}\left(T_{T P}\right)-\gamma_{l v}\left(T_{T P}\right) \approx 0.374
$$

The orientation averaged value of $\gamma_{s l}$, found in MD simulations $^{16}$ is $\gamma_{s l}^{M D}=0.360 \pm 0.02$ which agrees with our theoretical estimate within $3.8 \%$ accuracy. The values of interfacial free energy at the triple point resulting from the present model and found in MD simulations are summarized in Table I.

TABLE I: Triple point values of the interfacial free energy. Theory: present model; $M D-B G$ : MD simulations of Ref. ${ }^{17}$; $M D-D L$ : MD simulations of Ref. ${ }^{16}$

\begin{tabular}{cccc}
\hline & Theory & MD-BG & MD-DL \\
\hline$\gamma_{s v}$ & 1.134 & 1.16 & $\ldots$ \\
$\gamma_{l v}$ & 0.760 & 0.75 & $\ldots$ \\
$\gamma_{s l}$ & 0.374 & 0.35 & 0.360 \\
\hline
\end{tabular}

In conclusion, we proposed a closed form expression (17) for the solid-vapor interfacial free energy, based on the statistical mechanical perturbation approach. The model is applied to calculate $\gamma_{s v}$ for the LJ system along the sublimation line for temperatures not far from the triple point. Application of the model at the triple point itself yields interfacial free energies between all three coexisting phases. Model predictions are in good agreement with MD simulations.

\section{Acknowledgments}

Support of Twister BV is acknowledged.

\section{References}

1 Bonn, D.; Eggers, J.; Indekeu, J. et al. Wetting and Spreading. Rev. Mod. Phys. 2009, 81, 739-805.

2 Kalikmanov, V.I. Nucleation Theory; Springer: Dordrecht, 2013.

${ }^{3}$ Chattopadhyay, R. Surface Wear: Analysis, Treatment, and Prevention; ASM International: Materials Park, OH, 2001.

${ }^{4}$ Israelachvili, J.N. Intermolecular and Surface Forces; Academic Press: Amsterdam, 1991.

${ }^{5}$ Shuttleworth, R. The Surface Tension of Solids. Proc. Phys. Soc. London Sect. A 1950, 63, 444-448.

${ }^{6}$ Ketcham, W.M.; Hobbs, P.V. An Experimental Determination of the Surface Energies of Ice, Philos. Mag. 1969, 19, 1161-1173.

7 Ketcham, W.M.; Hobbs, P.V. In Physics of Ice; Riehl, N.; et al., Eds.), Plenum Press: N.Y., 1969; p.95.

8 Israelachvili, J.N. Forces Between Surfaces in Liquids. Adv. Colloid Interface Sci. 1982, 16, 31-47.

${ }^{9}$ Curtin, W.A. Density-Functional Theory of the SolidLiquid Interface. Phys. Rev. Lett. 1987, 59, 1228-1231.

10 Marr, D.W.; Gast, A.P. Planar Density-Functional Approach to the Solid-Fluid Interface of Simple Liquids. Phys. Rev. E 1993, 47, 1212-1221.

11 Ohnesorge, R.; Lowen, H.; Wagner, H. Density Functional
Theory of Crystal-Fluid Interfaces and Surface Melting. Phys. Rev. E 1994, 50, 4801-4810.

12 Davidchack, R.L.; Laird, B.B. Direct Calculation of the Hard-Sphere Crystal /Melt Interfacial Free Energy. Phys. Rev. Lett. 2000, 85, 4751-4754.

${ }^{13} \mathrm{Mu}$, Y.; Houk, A.; Song, X. Anisotropic Interfacial Free Energies of the Hard-Sphere Crystal-Melt Interfaces. $J$. Phys. Chem. B 2005, 109, 6500-6504.

14 Davidchack, R.L.; Laird, B.B. Crystal Structure and Interaction Dependence of the Crystal-Melt Interfacial Free Energy. Phys. Rev. Lett. 2005, 94, 086102.

15 Broughton, J.Q.; Gilmer, G.H. Molecular Dynamics Investigation of the CrystalFluid Interface. VI. Excess Surface Free Energies of CrystalLiquid Systems. J. Chem. Phys. 1986, 84, 5759-5768.

16 Davidchack, R.L.; Laird, B.B. Direct Calculation of the Crystal-Melt Interfacial Free Energies for Continuous Potentials: Application to the Lennard-Jones System. J. Chem. Phys. 2003, 118, 7651-7657.

17 Broughton, J.Q.; Gilmer, G.H. Surface Free Energy and Stress of a Lennard-Jones Crystal. Acta Metall. 1983, 31, 845-851.

18 Broughton, J.Q.; Gilmer, G.H. Molecular Dynamics Investigation of the CrystalFluid Interface. IV. Free Energies of Crystal-Vapor Systems. J. Chem. Phys. 1986, 84, 57415748. 
19 Kalikmanov, V.I. Statistical physics of fluids. Basic concepts and applications; Springer-Verlag: Berlin, 2001.

20 Smith, P.; Lynden-Bell, L.M. Determining Surface Free Energies of Crystals with Highly Disordered Surfaces from Simulation. Mol. Phys. 1999, 96, 1027-1032.

21 Modak, V.P.; Wyslouzil, B.E.; Singer, S.J. On the Determination of the Crystal-Vapor Surface Free Energy, and Why a Gaussian Expression Can Be Accurate for a System Far from Gaussian. J. Chem. Phys. 2016, 145, 054710.

${ }^{22}$ Foiles, S.M. Evaluation of Harmonic Methods for Calculating the Free Energy of Defects in Solids. Phys. Rev. B 1994, 49, 14930-14939.

${ }^{23}$ Frolov, T.; Mishin, Y. Temperature Dependence of the Surface Free Energy and Surface Stress: An Atomistic Calculation for $\mathrm{Cu}(110)$. Phys. Rev. B 2009, 79, 045430.

${ }^{24}$ Kirkwood, J.G.; Buff, F.P. The Statistical Mechanical Theory of Surface Tension. J. Chem. Phys. 1949, 17, 338343.

25 Schofield, P.; Henderson, J.R. Statistical Mechanics of Inhomogeneous Fluids. Proc. Phys. Soc. A 1982, 379, 231.

26 Rowlinson, J.S.; Widom, B. Molecular theory of capillarity; Clarendon Press: Oxford, 1982.

27 Kalikmanov, V.I.; Hofmans, G.C.J. The Perturbation Approach to the Statistical Theory of Surface Tension: New Analytical Results J. Phys.: Condensed Matter 1994, 6, 2207-2214

28 Weeks, D.; Chandler, D.; Andersen, H.C. Role of Repulsive Forces in Determining the Equilibrium Structure of Simple Liquids. J. Chem. Phys. 1971, 54, 5237-5247.

29 Hall, K.R. Another Hard-Sphere Equation of State. J. Chem. Phys. 1972, 57, 2252-2254.

30 Conway, J.H.; Sloane, N.J.A. Sphere Packings, Lattices, and Groups, 2nd ed.; Springer-Verlag: N.Y., 1993.

31 van der Hoef, M. Gas-Solid Coexistence of the LennardJones System, J. Chem. Phys. 2002, 117, 5092-5093.

32 Barroso, M.A.; Ferreira, A.L. Solid-Fluid Coexistence of the Lennard-Jones System From Absolute Free Energy Calculations. J. Chem. Phys. 2002, 116, 7145-7150.

33 Frenkel, D.; Ladd, A. New Monte Carlo Method to Compute the Free Energy of Arbitrary Solids. Application to the FCC and HCP Phases of Hard Spheres. J. Chem. Phys. 1984, 81, 3188-3193.

${ }^{34}$ Laird, B.B.; Davidchack, R.L. Direct Calculation of the Crystal-Melt Interfacial Free Energy via Molecular Dynamics Computer Simulation. J. Phys. Chem. B 2005, 109, 17802-17812.

35 Song, Y.; Mason E.A. Statistical-Mechanical Theory of a New Analytical Equation of State. J. Chem. Phys. 1989, $91,7840-7853$.

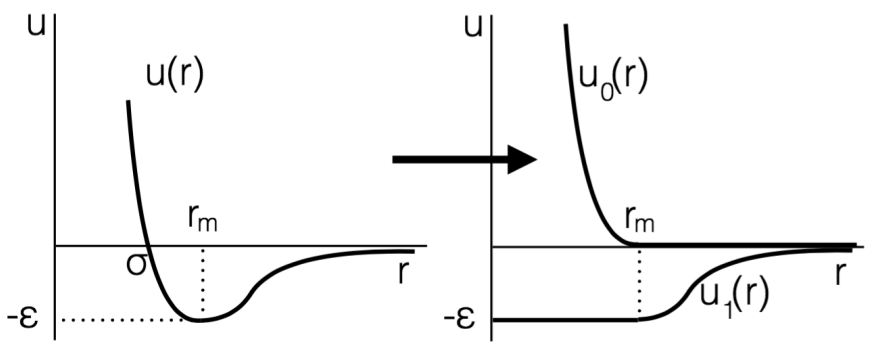

FIG. 4: TOC Graphic 